\title{
Front Matter: Volume 9786
}

, "Front Matter: Volume 9786," Proc. SPIE 9786, Medical Imaging 2016: Image-Guided Procedures, Robotic Interventions, and Modeling, 978601 (27 July 2016); doi: 10.1117/12.2240097

SPIE. Event: SPIE Medical Imaging, 2016, San Diego, California, United States 


\section{PROGRESS IN BIOMEDICAL OPTICS AND IMAGING \\ Vol. 17 No. 41}

\section{Medical Imaging 2016 Image-Guided Procedures, Robotic Interventions, and Modeling}

Robert J. Webster III

Ziv R. Yaniv

Editors

28 February-1 March 2016

San Diego, California, United States

Sponsored by

SPIE

Cosponsored by

Modus Medical Devices Inc. (Canada) - Bruker (United States) - Poco Graphite (United States) • imXPAD (France) • Siemens (Germany) • National Diagnostic Imaging (United States)

Cooperating Organizations

AAPM-American Association of Physicists in Medicine (United States) • APS-American Physiological Society (United States) - IFCARS—International Foundation for Computer Assisted Radiology and Surgery (Germany) - Medical Image Perception Society (United States) • Radiological Society of North America (United States) • Society for Imaging Informatics in Medicine (United States) • World Molecular Imaging Society The DICOM Standards Committee

Published by

SPIE

Volume 9786 
The papers in this volume were part of the technical conference cited on the cover and title page. Papers were selected and subject to review by the editors and conference program committee. Some conference presentations may not be available for publication. Additional papers and presentation recordings may be available online in the SPIE Digital Library at SPIEDigitallibrary.org.

The papers reflect the work and thoughts of the authors and are published herein as submitted. The publisher is not responsible for the validity of the information or for any outcomes resulting from reliance thereon.

Please use the following format to cite material from these proceedings:

Author(s), "Title of Paper," in Medical Imaging 2016: Image-Guided Procedures, Robotic Interventions, and Modeling, edited by Robert J. Webster III, Ziv R. Yaniv, Proceedings of SPIE Vol. 9786 (SPIE, Bellingham, WA, 2016) Six-digit Article CID Number.

ISSN: 1605-7422

ISSN: $2410-9045$ (electronic)

ISBN: 9781510600218

Published by

SPIE

P.O. Box 10, Bellingham, Washington 98227-0010 USA

Telephone +1 3606763290 (Pacific Time) · Fax +1 3606471445

SPIE.org

Copyright (C) 2016, Society of Photo-Optical Instrumentation Engineers.

Copying of material in this book for internal or personal use, or for the internal or personal use of specific clients, beyond the fair use provisions granted by the U.S. Copyright Law is authorized by SPIE subject to payment of copying fees. The Transactional Reporting Service base fee for this volume is $\$ 18.00$ per article (or portion thereof), which should be paid directly to the Copyright Clearance Center (CCC), 222 Rosewood Drive, Danvers, MA 01923. Payment may also be made electronically through CCC Online at copyright.com. Other copying for republication, resale, advertising or promotion, or any form of systematic or multiple reproduction of any material in this book is prohibited except with permission in writing from the publisher. The CCC fee code is $1605-7422 / 16 / \$ 18.00$.

Printed in the United States of America.

Publication of record for individual papers is online in the SPIE Digital Library.

\section{SPIE. DIGITAL}

SPIEDigitalLibrary.org

Paper Numbering: Proceedings of SPIE follow an e-First publication model. A unique citation identifier (CID) number is assigned to each article at the time of publication. Utilization of CIDs allows articles to be fully citable as soon as they are published online, and connects the same identifier to all online and print versions of the publication. SPIE uses a six-digit CID article numbering system structured as follows:

- The first four digits correspond to the SPIE volume number.

- The last two digits indicate publication order within the volume using a Base 36 numbering

system employing both numerals and letters. These two-number sets start with 00, 01, 02, 03, 04, 05, 06, 07, 08, 09, OA, OB ... 0Z, followed by 10-1Z, 20-2Z, etc. The CID Number appears on each page of the manuscript. 


\title{
Contents
}

\author{
xi Authors \\ xvii Conference Committee \\ xxi Introduction \\ xxiii 2016 Medical Imaging Award Recipients
}

\section{Part One}

\section{SESSION 1 CARDIAC PROCEDURES}

978602 Improved image guidance technique for minimally invasive mitral valve repair using real-time tracked 3D ultrasound [9786-1]

978603 Cognitive tools pipeline for assistance of mitral valve surgery [9786-2]

978604 Dynamic tracking of prosthetic valve motion and deformation from bi-plane x-ray views: feasibility study [9786-3]

978605 Classification of calcium in intravascular OCT images for the purpose of intervention planning [9786-4]

978606 Fusion of CTA and XA data using 3D centerline registration for plaque visualization during coronary intervention [9786-5]

\section{SESSION 2 SEGMENTATION AND 2D AND 3D REGISTRATION}

978607 Random walk based segmentation for the prostate on 3D transrectal ultrasound images [9786-6]

978608 Resection planning for robotic acoustic neuroma surgery [9786-7]

978609 Fat segmentation on chest CT images via fuzzy models [9786-8]

9786 OA Automatic masking for robust 3D-2D image registration in image-guided spine surgery [9786-9]

9786 OB Robust patella motion tracking using intensity-based 2D-3D registration on dynamic bi-plane fluoroscopy: towards quantitative assessment in MPFL reconstruction surgery [9786-10] 
9786 OC Fast generation of digitally reconstructed radiograph through an efficient preprocessing of ray attenuation values $[9786-11]$

\section{SESSION 3 SPINE AND PERCUTANEOUS PROCEDURES}

9786 OD Accurate biopsy-needle depth estimation in limited-angle tomography using multi-view geometry [9786-12]

$9786 \mathrm{OE}$ Automatic geometric rectification for patient registration in image-guided spinal surgery [9786-13]

9786 OF Real-time self-calibration of a tracked augmented reality display [9786-14]

9786 OG Clinical workflow for spinal curvature measurement with portable ultrasound [9786-15]

9786 OH MIND Demons for MR-to-CT deformable image registration in image-guided spine surgery (Robert F. Wagner All-Conference Best Student Paper Award) (Young Scientist Award) [9786-16]

\section{SESSION 4 ULTRASOUND IMAGE GUIDANCE: JOINT SESSION WITH CONFERENCES 9786 AND 9790}

9786 ol Automatic detection of a hand-held needle in ultrasound via phased-based analysis of the tremor motion [9786-17]

$97860 \mathrm{~J}$ Ultrasound to video registration using a bi-plane transrectal probe with photoacoustic markers [9786-18]

9786 OK Classification of prostate cancer grade using temporal ultrasound: in vivo feasibility study [9786-19]

\section{SESSION 5 REGISTRATION}

9786 OL Deformable registration of $x$-ray to MRI for post-implant dosimetry in prostate brachytherapy [9786-20]

9786 OM Evaluation of a $\mu \mathrm{CT}$-based electro-anatomical cochlear implant model [9786-21]

9786 ON Fast simulated annealing and adaptive Monte Carlo sampling based parameter optimization for dense optical-flow deformable image registration of 4DCT lung anatomy [9786-22]

978600 Automatic pose correction for image-guided nonhuman primate brain surgery planning [9786-23]

$9786 \mathrm{OP}$ Accurate tracking of tumor volume change during radiotherapy by СT-СBCT registration with intensity correction [9786-24] 
$97860 Q \quad$ Towards disparity joint upsampling for robust stereoscopic endoscopic scene reconstruction in robotic prostatectomy [9786-25]

9786 OR Endoscopes and robots for tight surgical spaces: use of precurved elastic elements to enhance curvature [9786-26]

9786 OS Disposable patient-mounted geared robot for image-guided needle insertion [9786-27]

9786 OT Comparison of portable and conventional ultrasound imaging in spinal curvature measurement [9786-90]

9786 OU Image-guided preoperative prediction of pyramidal tract side effect in deep brain stimulation (Young Scientist Award Runner Up) [9786-29]

9786 OV Rapid virtual stenting for intracranial aneurysms [9786-30]

\section{SESSION 7 TISSUE DEFORMATION AND MOTION}

9786 OW Surface driven biomechanical breast image registration [9786-31]

9786 0X Modeling and simulation of tumor-influenced high resolution real-time physics-based breast models for model-guided robotic interventions [9786-32]

9786 OY Accuracy of lesion boundary tracking in navigated breast tumor excision [9786-33]

9786 OZ Diaphragm motion characterization using chest motion data for biomechanics-based lung tumor tracking during EBRT [9786-34]

978610 Determination of surgical variables for a brain shift correction pipeline using an Android application [9786-35]

$978611 \quad$ Non-rigid point set registration of curves: registration of the superficial vessel centerlines of the brain [9786-36]

978612 A novel craniotomy simulation system for evaluation of stereo-pair reconstruction fidelity and tracking [9786-37]

\section{SESSION 8 INTRAOPERATIVE IMAGING AND VISUALIZATION}

978613 Biplane reconstruction and visualization of virtual endoscopic and fluoroscopic views for interventional device navigation [9786-38]

978614 Visual feedback mounted on surgical tool: proof of concept [9786-39]

978615 CT thermometry for cone-beam CT guided ablation [9786-40] 
978616 A computational model for estimating tumor margins in complementary tactile and 3D ultrasound images [9786-41]

978617 Freehand 3D-US reconstruction with robust visual tracking with application to ultrasound-augmented laparoscopy [9786-42]

\section{SESSION 9 ENDOSCOPY/LAPAROSCOPY}

978618 Superpixel-based structure classification for laparoscopic surgery [9786-43]

978619 Tissue classification for laparoscopic image understanding based on multispectral texture analysis [9786-44]

9786 1A Endoscopic feature tracking for augmented-reality assisted prosthesis selection in mitral valve repair [9786-45]

9786 1B Method for endobronchial video parsing [9786-46]

9786 1C Uncalibrated stereo rectification and disparity range stabilization: a comparison of different feature detectors [9786-47]

9786 ID Position-based adjustment of landmark-based correspondence finding in electromagnetic sensor-based colonoscope tracking method [9786-48]

\section{Part Two}

\section{SESSION 10 KEYNOTE AND NEW ROBOTIC APPLICATIONS}

$9786 \mathrm{IF}$ Toward automated cochlear implant insertion using tubular manipulators [9786-50]

9786 IG Increasing safety of a robotic system for inner ear surgery using probabilistic error modeling near vital anatomy (Young Scientist Award Runner Up) [9786-51]

\section{SESSION 11 PROSTATE PROCEDURES}

$9786 \mathrm{1H}$ A comparison of needle tip localization accuracy using 2D and 3D trans-rectal ultrasound for high-dose-rate prostate cancer brachytherapy treatment planning [9786-52]

9786 II An MRI guided system for prostate laser ablation with treatment planning and multi-planar temperature monitoring [9786-53]

9786 i J How does prostate biopsy guidance error impact pathologic cancer risk assessment? [9786-54]

9786 IK Impact of region contouring variability on image-based focal therapy evaluation [9786-55] 
9786 1L Structure Sensor for mobile markerless augmented reality [9786-56]

$97861 \mathrm{M} \quad$ Visual design and verification tool for collision-free dexterous patient specific neurosurgical instruments [9786-57]

9786 1N A web-based computer aided system for liver surgery planning: initial implementation on RayPlus [9786-58]

$978610 \quad$ Kinect based real-time position calibration for nasal endoscopic surgical navigation system [9786-59]

9786 IP An improved robust hand-eye calibration for endoscopy navigation system [9786-60]

$97861 Q \quad$ Towards robust specularity detection and inpainting in cardiac images [9786-61]

9786 IR Real-time mosaicing of fetoscopic videos using SIFT [9786-62]

9786 is Multiple video sequences synchronization during minimally invasive surgery [9786-63]

9786 IT Visualization framework for colonoscopy videos [9786-64]

$97861 \mathrm{U} \quad$ HPC enabled real-time remote processing of laparoscopic surgery [9786-65]

$97861 \mathrm{~V}$ Content-based retrieval in videos from laparoscopic surgery [9786-66]

9786 1W Cost-effective surgical registration using consumer depth cameras [9786-67]

$97861 \mathrm{X} \quad$ Exploring the effects of dimensionality reduction in deep networks for force estimation in robotic-assisted surgery [9786-68]

$97861 \mathrm{Y}$ Current sensing for navigated electrosurgery: proof of concept [9786-69]

$97861 \mathrm{Z}$ Characterization of a phantom setup for breast conserving cancer surgery [9786-70]

978620 Image-guided intracranial cannula placement for awake in vivo microdialysis in nonhuman primates [9786-71]

$978621 \quad$ Patch-based label fusion for automatic multi-atlas-based prostate segmentation in MR images (Cum Laude Poster Award) [9786-72]

978622 Phantom-based ground-truth generation for cerebral vessel segmentation and pulsatile deformation analysis [9786-73]

978623 A general approach to liver lesion segmentation in CT images [9786-74]

978624 A comparison study of atlas-based 3D cardiac MRI segmentation: global versus global and local transformations [9786-75]

978625 Surface mesh to voxel data registration for patient-specific anatomical modeling [9786-76] 
978626 Estimation of line-based target registration error [9786-77]

978627 A MRI-CT prostate registration using sparse representation technique [9786-78]

978629 Fusion of cone-beam CT and 3D photographic images for soft tissue simulation in maxillofacial surgery [9786-80]

9786 2A Image updating for brain deformation compensation in fumor resection [9786-81]

9786 2B A fully automatic image-to-world registration method for image-guided procedure with intraoperative imaging updates [9786-82]

9786 2C Optimal atlas construction through hierarchical image registration [9786-83]

9786 2D Single slice US-MRI registration for neurosurgical MRI-guided US [9786-84]

9786 2E Rapidly-steered single-element ultrasound for real-time volumetric imaging and guidance [9786-85]

$97862 \mathrm{~F} \quad$ Investigation of permanent magnets in low-cost position tracking [9786-86]

$97862 \mathrm{G} \quad$ Image-guided endobronchial ultrasound [9786-87]

$97862 \mathrm{H} \quad$ A motorized ultrasound system for MRI-ultrasound fusion guided prostatectomy [9786-88]

97862 Visualization of hepatic arteries with 3D ultrasound during intra-arterial therapies [9786-89]

9786 2J 3D shape tracking of minimally invasive medical instruments using optical frequency domain reflectometry [9786-91]

9786 2K Measurement of electromagnetic tracking error in a navigated breast surgery setup [9786-92]

$97862 \mathrm{~L} \quad$ Image-guided navigation surgery for pelvic malignancies using electromagnetic tracking [9786-93]

$97862 \mathrm{M} \quad$ Feasibility of tracked electrodes for use in epilepsy surgery [9786-94]

9786 2N 4D cone-beam CT imaging for guidance in radiation therapy: setup verification by use of implanted fiducial markers [9786-95]

978620 Effects of voxelization on dose volume histogram accuracy [9786-96]

$97862 \mathrm{P} \quad$ Partition-based acquisition model for speed up navigated beta-probe surface imaging [9786-97]

$97862 Q \quad$ Stent enhancement in digital x-ray fluoroscopy using an adaptive feature enhancement filter [9786-98]

$97862 R \quad$ Evaluation of left ventricular scar identification from contrast enhanced magnetic resonance imaging for guidance of ventricular catheter ablation therapy [9786-99] 
978625 Interactive visualization for scar transmurality in cardiac resynchronization therapy [9786-100]

$97862 \mathrm{~A} \quad$ A robust automated left ventricle region of interest localization technique using a cardiac cine MRI atlas [9786-101]

$97862 \mathrm{U}$ Classification of coronary artery tissues using optical coherence tomography imaging in Kawasaki disease [9786-102] 
Proc. of SPIE Vol. $9786978601-10$

Downloaded From: https://www.spiedigitallibrary.org/conference-proceedings-of-spie on 26 Apr 2023 Terms of Use: https://www.spiedigitallibrary.org/terms-of-use 


\section{Authors}

Numbers in the index correspond to the last two digits of the six-digit citation identifier (CID) article numbering system used in Proceedings of SPIE. The first four digits reflect the volume number. Base 36 numbering is employed for the last two digits and indicates the order of articles within the volume. Numbers start with 00, 01, 02, 03, 04, 05, 06, 07, 08, 09, OA, OB...0Z, followed by 10-1Z, 20-2Z, etc.

Abdolmanafi, Atefeh, $2 \mathrm{U}$

Abi-Jaoudeh, Nadine, 15

Abolmaesumi, Purang, OK

Agarwal, Harsh, 11

Ahmed, Hashim U., 1K

Alderliesten, Tanja, 2N

Alsaleh, Samar M., 1Q, IX

Anderson, Ryan, 2F

Aviles, Angelica I., 1Q, 1X

Badoual, Anaiis, 2

Bagchi, Ansuman, 00, 20

Bainbridge, Daniel, 02

Baksh, Aidan, 2K

Balachandran, Ramya, 08, IG

Barratt, Dean C., IK

Bascom, Rebecca, 2G

Baum, Zachary, OF, 2K

Baumgarten, C., OU

Baxter, John S. H., 2D

Beecks, Christian, IV

Behar, Jonathan M., $2 S$

Beigi, Parmida, 0 I

Bel, Arjan, 2N

Belhaoua, Abdelkrim, is

Ben-Zikri, Yehuda Kfir, 24, 2T

Bernardo, Marcelino, 11

Bezerra, Hiram G., 05

Bigot, Alexandre, 21

Blachon, Gregoire S., $1 G$

Boctor, Emad M., OJ

Bodani, Vivek, $1 \mathrm{M}$

Bodenstedt, Sebastian, 18

Bone, Ashleigh, 00, 20

Borschneck, Daniel, 0G, OT

Brinkmann, Benjamin, 2M

Brost, Alexander, 25

Buelow, Thomas, OW

Burgner-Kahrs, Jessica, IF

Bux, R., $1 \mathrm{~L}$

Byrnes, Patrick D., 1B, 2 G

Cai, Yunliang, OE

Cakir, Ahmet, OM

Cao, Li, 23

Carter, K., 14, 1Y

Casals, Alicia, 1Q, IX

Chadebecq, François, IR

Chadwell, Jacob T., $1 \mathrm{Z}$

Chang, Silvia, OK

Cheirsilp, Ronnarit, 2G
Chen, Antong, 00, 20

Chen, Danyang, OV

Chen, Elvis C. S., 17, 26

Chen, Zihe, oV

Cheng, Alexis, oJ

Chin, Joseph L., $1 \mathrm{~J}$

Choyke, Peter, 11

Christie, Jason, 09

Chu, Yakui, 10

Chung, Soyoung, 29

Clancy, Neil T., 19

Clements, Logan W., 10, 12

Collins, Jarrod A., 12

Conley, Rebekah H., 10, 12, 1 Z

Cool, Derek W., $1 \mathrm{~J}$

Corso, Jason J., OV

Curran, Walter J., 21, 27

Daga, Pankaj, IR

Dahdah, Nagib, 2U

Dangi, Shusil, 24

Daryanani, Aditya, 24

David, Anna L., IR

Dawant, Benoit M., 08, 0M, 12

de Oliveira, Júlia E. E., 25

De Silva, T., OA, $\mathrm{OH}$

De Simone, Raffaele, 03, 1A

de With, Peter H. N., OD

Deprest, Jan, $1 \mathrm{R}$

Deserno, Thomas M., 25

DeStefano, Zachary, 15

Dijkstra, Jouke, 06

Dillmann, Rüdiger, 18

Dillon, Neal P., $1 G$

Dogdas, Belma, 00, 20

Donaldson, Ian A., $1 \mathrm{~K}$

Dou, Tai H., ON

Drake, James, $1 \mathrm{M}$

D'Souza, David, $1 \mathrm{H}$

Duffy, Edward, $1 \mathrm{U}$

Duong, LuC, $2 U$

Dwyer, George, IR

Eastwood, Kyle, 1M

Edirisinghe, Chandima, $1 \mathrm{H}$

Eiben, Björn, OW

Elson, Daniel S., 19

Engel, C. Jay, OY, 14, 2K

Engelhardt, Sandy, 03, $1 \mathrm{~A}$

Escoto, Abelardo, 16

Esnault, Matthieu, OB 
Evans, Linton T., OE

Fahn, Bernhard, 2S

Fan, Jingfan, 10

Fan, Xiaoyao, $0 \mathrm{E}, 2 \mathrm{~A}$

Fangerau, M., $1 \mathrm{~L}$

Fei, Baowei, 07

Fenster, Aaron, $\mathrm{OK}, 1 \mathrm{H}, 1 \mathrm{~J}$

Fetzer, Andreas, 03

Fichtinger, Gabor, OF, OG, OT, OY, 14, 1M, 1Y, 2F, $2 \mathrm{~K}, 2 \mathrm{O}$

Fitzpatrick, J. Michael, $1 G$

Franz, A. M., 1L

Furukawa, Kazuhiro, 1D

Gaed, Mena, OK, 1J

Gaede, Stewart, $\mathrm{OZ}$

Gauvin, Gabrielle, OY, 14, 2K

Gérard, Maxime, 21

Ghafurian, Soheil, 0C, 00, 20

Ghavidel, Sahar, OK

Gibson, Eli, OK, 1 J, 1K

Giessler, Paul, 25

Glossop, Neil, 11

Gómez, José A., OK, $1 \mathrm{~J}$

Görtler, Jochen, 18

Goto, Hidemi, 1D

Granna, Josephine, $1 F$

Grevera, George J., 2C

Grupp, Robert, OB

Guo, Rongrong, 07

Haegelen, C., OU

Hahn, James, $1 Q$

Hanson, Dennis, $2 M$

Harish, Vinyas, 2K

Hartov, Alex, 2A

Hashtrudi-Zaad, Keyvan, 2F

Hasse, Katelyn, OX

Hata, Nobuhiko, OS

Hatt, Charles R., 04

Hawkes, David J., OW

$\mathrm{He}$, Fei, IP

$\mathrm{He}$, Wei, $1 \mathrm{P}$

Heffernan, Emily, OY

Heim, E., 1L

Herrera, Luis Carlos Garcia-Peraza, IR

Herrler, Andreas, 25

Hess, Maggie, $1 \mathrm{M}$

Heuveline, Vincent, 03

Higgins, William E., 1B, $2 \mathrm{G}$

Hines, Catherine D. G., 00, 20

Hipwell, John H., OW

Hirooka, Yoshiki, 1D

Holden, M., 14

Holmes, David, III, 2M

Holton, Leslie, 2M

Hong, Helen, 29

Hoover, Douglas A., $1 \mathrm{H}$

Hrinivich, W. Thomas, $1 \mathrm{H}$

Hristov, Dmitre, 2E

$\mathrm{HU}$, Yipeng, 1K

Huang, Lidong, 23
Hulshof, Maarten C. C. M., 2N

Illies, Till, 22

Imani, Farhad, OK

Iszatt, Justin, 19

Izard, Ryan, $1 \mathrm{U}$

Jani, Ashesh B., 21, 27

Jannin, Pierre, OU, 2P

Jayarathne, Uditha L., OQ, 17, 1C

Ji, Songbai, OE, 2A

Jiang, Yuhao, $2 Q$

Jiang, Zhengang, $1 \mathrm{P}$

Jin, Peng, $2 \mathrm{~N}$

Johnen, W., $1 \mathrm{~L}$

Johnson, Colena, 00, 20

Johnson, S. B., 2R

Kabus, Sven, OW

Kadoury, Samuel, 2I, 2J

Kaila, Gaurav, 06

Kang, Hyun Jae, OJ

Kang, Jin U., OJ

Kang, Kumsok, IP

Kanti Mandal, Koushik, 2J

Karami, Elham, $\mathrm{OZ}$

Karck, Matthias, 1 A

Karim, Rashed, 2S

Kashyap, Raman, 2J

Kato, Takahisa, OS

Kaufman, Arie, $1 \mathrm{~T}$

Kempston, Michael, OG, OT

Kenngott, Hannes, 18, 19

Keshtgar, M., OW

Keszei, András, 25

Ketcha, M. D., OA

Khallaghi, Siavash, OK

Khan, Ali R., 2D

Khanna, A. J., $\mathrm{OH}$

Khojaste, Amir, OK

Kiess, Ana P., OP

Kilgus, T., 1L

Kim, Joojin, 29

Kitasaka, Takayuki, 1D

Kitslaar, Pieter, 06

Kleinszig, G., OA, OH

Kolb, Silvio, 1A

Kondo, Hiroaki, 1D

Kording, Fabian, 22

Kosugi, Shinichi, OB

Krebs, Alexandre, 1s

Kuhlengel, Trevor, 2G

Kuhlmann, Koert, 2L

Kupelian, Patrick, ON

Kwartowitz, David M., $1 \mathrm{U}$

Labadie, Robert F., 08

Lasso, Andras, OF, OY, 14, 1M, 1Y, 2F, 2K, 20

Lederer, David J., 09

Lee, Junghoon, OL, OP

Lee, Ting-Yim, $0 Z$

Lehmann, H. I., $2 R$

Lelieveldt, Boudewijn, 06

Lenarz, Thomas, IF 
Leveridge, Michael, OK

Li, Kang, OC

Li, Ming, 15

Li, Senhu, 2B

Li, Tianhong, $1 \mathrm{~N}$

Li, Yanfang, IP

Linder, Bence, $1 \mathrm{M}$

Linte, Cristian A., 24, $2 T$

Liu, Tian, 21, 27

Liu, Xiabi, 07

Lodge, Kenneth, 00

Lollis, S. Scott, OE

Looi, Thomas, IM

Loranger, Sebastien, 2J

Lorenz, Cristian, OW

Lubbers, Laura S., 00, 20

Luo, Ming, $1 \mathrm{~N}$

Luo, Xiongbiao, OQ, 1C

Lux, Mathias, $1 \mathrm{~V}$

Ma, Burton, 26

Ma, Ling, 07

Ma, Shaodong, 10

Maier-Hein, Lena, 19, 1L

Majdani, Omid, IF

Maleshkova, Maria, 03

Malrain, C., OU

Mao, Hui, 21, 27

Marescaux, Jacques, is

Marreiros, Filipe M. M., 11

Martin, Peter R., $1 \mathrm{~J}$

Master, Viraj V., 07

Mayer, Benjamin, 19

McBrayer, Kepra L., 08

McLeod, A. Jonathan, OQ, IC

Meinzer, Hans-Peter, IA

Meng, Hui, OV

Meszoely, Ingrid M., $1 \mathrm{Z}$

Metaxas, Dimitris N., OC

Miao, Yu, IP

Michaud, François, 2I

Michener, Maria, 20

Miga, Michael I., 10, 12, 12

Min, Yugang, ON

Mirza, Sohail K., OE

Mistretta, Charles A., 13

Mitchell, Jason E., 1G

Miyahara, Ryoji, 1D

Monge, Frédéric, 2P

Montgomery, Tamara O., 20

Montreuil, Jacques, $1 \mathrm{H}$

Moore, John, 02, 17

Moreau, Johan, is

Mori, Kensaku, 1D, 1P

Morin, Evelyn, OY, 14, 1Y

Mountney, Peter, $2 S$

Mousavi, Parvin, OK

Moussa, Madeleine, OK, $1 \mathrm{~J}$

Müller, M., $1 \mathrm{~L}$

Müller-Stich, Beat Peter, 18

Nadeem, Saad, $1 T$
Naish, Michael D., 16

Navab, Nassir, 1D, 2P

Negussie, Ayele, 11

Neylon, John, ON, OX

$\mathrm{Ng}$, Gary C., Ol

Nguyen, Thien-Dang, IF

Nieh, Peter T., 07

Nijkamp, Jasper, 2L

Noble, Jack H., 08, 0M

Nolden, Marco, 03

Oda, Masahiro, ID

Odhner, Dewey, 23, 2C

Olson, Jonathan D., OE, 2A

O'Malley, Stacey, $0 \mathrm{O}$

Otake, Yoshito, OB

Ourselin, Sebastien, IR

Packer, D. L., 2R

Paliwal, Nikhil, OV

Panayiotou, Maria, 25

Pardasani, Utsav, 2D

Parent, Francois, 2J

Park, Seyoun, OL, OP

Partanen, Ari, 11

Patel, Rajni V., 16

Paulsen, Keith D., OE, 2A

Pautler, Stephen E., OQ, 1C, 1J

Pednekar, Gargi, 09

Penicka, Martin, 06

Peters, Terry M., 02, 0Q, 17, 1C, 26, 2D

Pezeshki, Padina, OY, 14

Philipp, Patrick, 03

Pinter, Csaba, 20

Pinto, Peter, 11, 2H

Plishker, William, OP

Potter, Michael, 1W

Prabhu, David, 05

Prasad, Arpan Suravi, $2 U$

Prince, J. L., $\mathrm{OH}$

Quon, Harry, OP

Radoux, Jean-Pierre, is

Rankin, Adam, 02

Rau, Thomas S., $1 \mathrm{~F}$

Raval, Amish N., 04

Ray, Soumya, 05

Razavi, Reza, 25

Reaungamornrat, S., $\mathrm{OH}$

Reiml, Sabrina, $2 S$

Remirez, Andria A., OR

Renger, John, 00, 20

Rettinger, Achim, 03

Rettmann, M. E., 2R

Rhode, Kawal S., $2 S$

Rinaldi, Christopher A., 2S

Robb, Richard, $2 \mathrm{M}$

Roberts, David W., OE, 2A

Robinson, Adam, OP

Rohling, Robert, 0 I

Ronaghi, Zahra, $1 \mathrm{U}$

Rossi, Peter J., 21, 27

Rossitti, Sandro, 11 
Rudan, John, OY, 14, 2K

Ruers, Theo, 2L

Salcudean, Septimiu E., Ol

Salisbury, Kenneth, 2E

Samani, Abbas, $0 Z$

Santhanam, Anand P., ON, OX

Sapra, Karan, IU

Säring, Dennis, 22

Sarkar, Saradwata, 07

Sarment, David, 2B

Sato, Yoshinobu, OB

Sauleau, P., OU

Schafer, Sebastian, 13

Schetelig, Daniel, 22

Schlacta, Christopher M., OQ, IC

Schlosser, Jeffrey, 2E

Schoch, Nicolai, 03

Schoeffmann, Klaus, IV

Schuster, David M., 07

Sedlacik, Jan, 22

Seidl, Thomas, $1 \mathrm{~V}$

Seifabadi, Reza, 11, 2H

Shah, Taimur T., 1K

Shakir, Dzhoshkun I., 1R, 2P

Shalev, Ronny, 05

Shamsil, Arefin, 16

Shekhar, Raj, OP

Shen, Colette, OP

Sheng, Ke, OX

Shi, Weili, IP

Siebold, Michael A., $1 G$

Siemens, D. Robert, OK

Siewerdsen, J. H., OA, OH

Smedby, Örjan, 11

Smith, Melissa C., IU

Sobrevilla, Pilar, 1Q, 1X

Solek, Roman, 2E

Song, Danny Y., OL

Sonke, Jan-Jakob, $2 \mathrm{~L}$

Soulez, Gilles, 2l

Speidel, Michael A., 04

Speidel, Stefanie, 18

Stauber, Mark, 2E

Stock, Christian, 19

Stoyanov, Danail, $1 R$

Strother, Charles M., 13

Studer, Rudi, 03

Subramanian, Janani Rajan, 09

Summers, Ronald M., 15

Sun, Zhi, IN

Sunderland, Kyle, 20

Surry, Kathleen, $1 \mathrm{H}$

Tabanfar, Reza, OG, OT

Tan, Virak, OC

Tang, An, 2l

Taylor, Russell H., OJ

Tella, Marcel, $1 R$

Thomas, David, ON

Thompson, Reid C., 10, 12

Tian, Zhiqiang, 07
Tong, Yubing, 09, 23

Torigian, Drew A., 09, 23, 2C

Toth, Daniel, $2 S$

Toth, Jennifer, 2G

Tu, Shengxian, 06

Turkbey, Baris, 11

Udupa, Jayaram K., 09, 23, 2C

Uneri, A., $\mathrm{OA}, \mathrm{OH}$

Ungi, Tamas, OF, OG, OT, OY, 14, 1Y, 2K

Uslaner, Jason M., 00, 20

Uysal, Merih Seran, IV

van der Sommen, Fons, OD

van Wieringen, Niek, $2 \mathrm{~N}$

Vaughan, Thomas, OY, 14

Vavourakis, Vasileios, $0 \mathrm{~W}$

Venkataraman, Rajesh, 07

Vercauteren, Tom, IR

Vijayan, Rohan, 10

Vogt, S., OA, OH

Volovyk, Mykola, 03

Waechter, Julien, IS

Wagner, Martin G., 04, 13

Wagner, Martin, 18, 19

Wang, Chunliang, 11

Wang, Kuang-Ching, 14

Wang, Xiangyu, OV

Wang, Yongtian, 10

Wanna, George B., 08

Ward, Aaron D., OK, $1 \mathrm{~J}$

Watanabe Fernandes, Eric Hideki, 2J

Watkins, Charles, OS

Webster, Robert J., III, OR, IG

Weller, Tobias, 03

Werner, René, 22

Western, Craig, 2E

Williams, Norman R., OW

Wilson, David L., 05

Winkelmann, Christopher T., 00, 20

Wirkert, Sebastian J., 19

Wolf, Ivo, 03, 1A

Wolinsky, J.-P., OA, OH

Wong, Eugene, $1 \mathrm{H}$

Wong, John, OP

Wood, Bradford J., 15, 1I, 2H

Worrell, Gregory, 2M

Wu, Caiyun, 09

Xiang, Jianping, OV

Xie, Qingguo, $1 \mathrm{~N}$

$X U$, Jinhui, OV

$\mathrm{XU}$, Sheng, 1I, 2H

Yan, Christina, OG, OT

Yan, Fei, IP

Yang, Huamin, IP

Yang, Jian, 10

Yang, Xiaochen, 12

Yang, Xiaofeng, 21, 27

Yaniv, Ziv, IW

Yao, Jianhua, 15

Yen, K., 1L

Yuan, Rong, iN 
Zachary, Josey, $2 Q$

Zang, Xiaonan, $2 \mathrm{G}$

Zariwala, Hatim A., 00, 20

Zhang, Haichong K., OJ

Zhang, Huimao, $1 \mathrm{P}$

Zhang, Yan, 19

Zhao, Liang, OV

Zhao, Y., OU

Zinger, Sveta, OD

Proc. of SPIE Vol. $9786978601-15$

Downloaded From: https://www.spiedigitallibrary.org/conference-proceedings-of-spie on 26 Apr 2023 Terms of Use: https://www.spiedigitallibrary.org/terms-of-use 
Proc. of SPIE Vol. $9786978601-16$

Downloaded From: https://www.spiedigitallibrary.org/conference-proceedings-of-spie on 26 Apr 2023 Terms of Use: https://www.spiedigitallibrary.org/terms-of-use 


\title{
Conference Committee
}

\author{
Symposium Chairs
}

Steven C. Horii, The University of Pennsylvania Health System

(United States)

Berkman Sahiner, U.S. Food and Drug Administration (United States)

Conference Chairs

Robert J. Webster III, Vanderbilt University (United States)

Ziv R. Yaniv, U.S. National Library of Medicine (United States)

\section{Conference Program Committee}

Purang Abolmaesumi, The University of British Columbia (Canada)

Wolfgang Birkfellner, Medizinische Universität Wien (Austria)

Sandrine de Ribaupierre, Western University (Canada)

Baowei Fei, Emory University (United States)

Gabor Fichtinger, Queen's University (Canada)

George J. Grevera, Saint Joseph's University (United States)

David J. Hawkes, University College London (United Kingdom)

David R. Haynor, University of Washington (United States)

William E. Higgins, The Pennsylvania State University (United States)

David R. Holmes III, Mayo Clinic (United States)

Pierre Jannin, Université de Rennes 1 (France)

David M. Kwartowitz, Clemson University (United States)

Cristian A. Linte, Rochester Institute of Technology (United States)

Lena Maier-Hein, Deutsches Krebsforschungszentrum (Germany)

Michael I. Miga, Vanderbilt University (United States)

Kensaku Mori, Nagoya University (Japan)

Parvin Mousavi, Queen's University (Canada)

Maryam E. Rettmann, Mayo Clinic (United States)

Frank Saver, Siemens Corporation (United States)

Eric J. Seibel, University of Washington (United States)

Guy Shechter, Philips Healthcare (United States)

Amber L. Simpson, Memorial Sloan Kettering Cancer Center

(United States)

Stefanie Speidel, Karlsruher Institut für Technologie (Germany)

Andrew D. Wiles, Northern Digital Inc. (Canada)

Ivo Wolf, Hochschule Mannheim (Germany) 


\section{Session Chairs}

1 Cardiac Procedures

Maryam E. Rettmann, Mayo Clinic (United States)

David R. Holmes III, Mayo Clinic (United States)

2 Segmentation and 2D and 3D Registration

David R. Haynor, University of Washington (United States)

Baowei Fei, Emory University (United States)

3 Spine and Percutaneous Procedures

Gabor Fichtinger, Queen's University (Canada)

David J. Hawkes, University College London (United Kingdom)

$4 \quad$ Ultrasound Image Guidance

Joint Session with Conferences 9786 and 9790

Parvin Mousavi, Queen's University (Canada)

Brecht Heyde, KU Leuven (Belgium) and Duke University (United States)

5 Registration

Lena Maier-Hein, Deutsches Krebsforschungszentrum (Germany)

Stefanie Speidel, Karlsruher Institut für Technologie (Germany)

6 Robotic Systems and Treatment Planning

David M. Kwartowitz, Clemson University (United States)

Cristian A. Linte, Rochester Institute of Technology (United States)

7 Tissue Deformation and Motion

Michael I. Miga, Vanderbilt University (United States)

Pierre Jannin, Université de Rennes 1 (France)

8 Intraoperative Imaging and Visualization

George J. Grevera, Saint Joseph's University (United States)

Amber L. Simpson, Memorial Sloan Kettering Cancer Center (United States)

9 Endoscopy/Laparoscopy

Kensaku Mori, Nagoya University (Japan)

William E. Higgins, The Pennsylvania State University (United States)

10 Keynote and New Robotic Applications

Robert J. Webster III, Vanderbilt University (United States)

Ziv Yaniv, U.S. National Library of Medicine (United States) 
11 Prostate Procedures

Purang Abolmaesumi, The University of British Columbia (Canada) Frank Sauer, Siemens Corporation (United States)

\section{Session Workshop Chair}

Sensing Challenges and Prospects in Miniaturizing Surgical Robots and Tools

Jessica Burgner-Kahrs, Leibniz Universität Hannover (Germany)

Interventional Procedures: Emerging Technologies and Clinical Applications

Ziv R. Yaniv, U.S. National Library of Medicine (United States) 
Proc. of SPIE Vol. $9786978601-20$

Downloaded From: https://www.spiedigitallibrary.org/conference-proceedings-of-spie on 26 Apr 2023 Terms of Use: https://www.spiedigitallibrary.org/terms-of-use 


\section{Introduction}

Welcome to the 2016 edition of the SPIE Image-Guided Procedures, Robotic Interventions, and Modeling conference proceedings. This year we received 113 abstract submissions and accepted 101 as full manuscripts which were presented at the conference during the oral and poster sessions.

The keynote presentation by Kenneth Goldberg from the University of California Berkeley was outstanding. The title of the talk was Robot-Assisted Tumor Resection: Palpation, Incision, Debridement and Adhesive Closure. Prof. Goldberg presented a fascinating view on the future of automation in robotic surgery. He described new tooling and learning algorithms to facilitate supervised automation of surgical subtasks. In particular, he described new approaches to palpation, dissection, retraction, debridement, and adhesive closure.

This year we held two parallel workshops; Sensing Challenges and Prospects in Miniaturizing Surgical Robots and Tools, and a joint workshop with the physics conference Interventional Procedures: Emerging Technologies and Clinical Applications.

Jessica Burgner-Kahrs organized the Sensing Challenges and Prospects in Miniaturizing Surgical Robots and Tools workshop. It featured three presentations on cutting edge research in robotics and sensing technologies. First, Jenny Dankelman from TU Delft in the Netherlands spoke about numerous medical devices being developed at Delft, including steerable needles, robotic catheters, and hand-held laparoscopic instruments. Next, Dan Popa of the University of Louisville spoke about sensors and the micro-manufacturing technologies used to create them. Lastly, Robert Webster of Vanderbilt University spoke about the need to consider sensing, design, and motion planning simultaneously in order to create optimal flexible robots, such as concentric tube robots. A major theme that emerged from the workshop was the need to integrate sensing systems into the material of the robot. Another was the need for many different kinds of sensors, including those measuring position, orientation, force, strain, contact, temperature for various surgical applications.

The joint workshop with the Conference 9790 was well attended and included talks from leading researchers in the field. Terry Peters from Robarts Research Institute described Imaging Systems for Minimally-Invasive Surgery. Jeffrey Siewerdsen from Johns Hopkins University talked about Interventional Imaging Technologies for Therapy Guidance. Kullervo Hynynen from Sunnybrook Health Sciences Center described Applications of Non-Invasive Surgery and Targeted Therapeutics, and Puneet Sharma from Siemens Healthcare talked about Technology Transfer of Interventional Imaging Technologies to the Clinic. 
This year's Young Scientist Award went to Sureerat Reaungamornrat from Johns Hopkins University (United States) for her paper titled MIND Demons for MR-to-CT deformable image registration in image-guided spine surgery. She also received the Robert F. Wagner All-Conference Best Student Paper Award for this work. This year we had two co-runners up for the Young Scientist Award, Clement Baumgarten from the University of Rennes I (France) for his paper titled Imageguided preoperative prediction of pyramidal tract side effect in deep brain stimulation and Neal Dillon form Vanderbilt University (United States) for his paper titled Increasing safety of a robotic system for inner ear surgery using probabilistic error modeling near vital anatomy. The winner of Best Poster Award this year was Xiaofeng Yang from Emory University (United States) and the two honorable mention awardees were Maggie Hess from Queen's University (Canada) and Burton Ma from York University (Canada). We would like to thank Siemens Healthcare for sponsoring the Young Scientist Award prizes and Northern Digital Inc. for sponsoring the prizes for our Best Poster Awards.

We are grateful to all of our committee members for their help in reviewing abstracts, evaluating student papers, and judging posters. Their commitment enables us to maintain the high scientific standards of our conference. We also recognize the outstanding service of Ziv Yaniv from the National Institutes of Health as chair of the conference; he has completed his term and is resuming his role as a committee member. Baowei Fai from Emory University is our new cochair, and will begin his four year term next year.

Finally, we would like to thank all the attendees who gave talks, presented posters, and actively participated in the meeting. The success of the conference is in no small part due to you. Next year, the conference will take place in Orlando, Florida. We look forward to seeing you there for another great conference! 


\section{Medical Imaging Award Recipients}

\section{Robert F. Wagner Best Student Paper Award}

Robert F. Wagner was an active scientist in the SPIE Medical Imaging meeting, starting with the first meeting in 1972 and continuing throughout his career. He ensured that the $\mathrm{BRH}$, and subsequently the $\mathrm{CDRH}$, was a sponsor for the early and subsequent Medical Imaging meetings, helping to launch and ensure the historical success of the meeting. The Robert $F$. Wagner All-Conference Best Student Paper Award (established 2014) is acknowledgment of his many important contributions to the Medical Imaging meeting and his many important advances to the field of medical imaging.

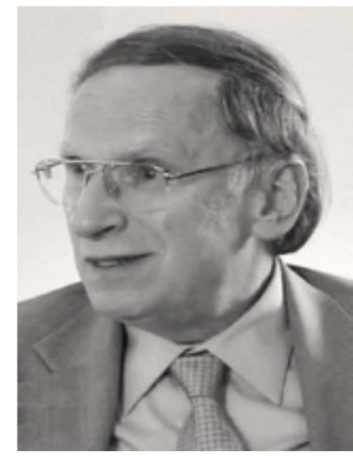

This award is cosponsored by:

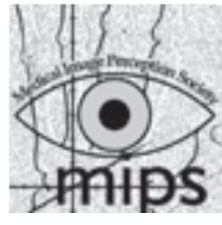

The Medical Image Perception Society

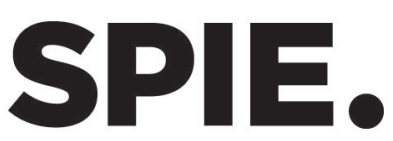

\section{Recipients:}

First Place: MIND Demons for MR-to-CT deformable image registration in image-guided spine surgery (9786-16)

S. Reaungamornrat, T. De Silva, A. Uneri, Johns Hopkins Univ. (United States), J.-P. Wolinsky, Johns Hopkins Hospital (United States), A. J. Khanna, Johns Hopkins Health Care \& Surgery Ctr. (United States), G. Kleinszig, S. Vogt, Siemens Healthcare (Germany), J. L. Prince, J. H. Siewerdsen, Johns Hopkins Univ. (United States)

Second Place: Design, fabrication, and implementation of voxel-based 3D printed textured phantoms for task-based image quality assessment in CT (9783-76)

Justin Solomon, Duke Univ. School of Medicine (United States), Alexandre Ba, Institut Univ. de Radiophysique Appliquée (Switzerland), Andrew Diao, Duke Univ. (United States), Joseph Lo, Elianna Bier, Duke Univ. School of Medicine (United States), François Bochud, Institut Univ. de Radiophysique Appliquée (Switzerland), Michael Gehm, Duke Univ. (United States), Ehsan Samei, Duke Univ. School of Medicine (United States)

\section{Conference 9786 Awards}

\section{Young Scientist Awards sponsored by Siemens}

First Place: MIND Demons for MR-to-CT deformable image registration in image-guided spine surgery (9786-16)

S. Reaungamornrat, T. De Silva, A. Uneri, Johns Hopkins Univ. (United States), J.-P. Wolinsky, Johns Hopkins Hospital (United States), A. J. Khanna, Johns Hopkins Health Care \& Surgery Ctr. (United States), G. Kleinszig, S. Vogt, Siemens Healthcare (Germany), J. L. Prince, J. H. Siewerdsen, Johns Hopkins Univ. (United States) 
Runner Up: Image-guided preoperative prediction of pyramidal tract side effect in deep brain stimulation (9786-29)

C. Baumgarten, Y. Zhao, INSERM (France), LTSI, Univ. de Rennes 1 (France); P. Sauleau, C. Malrain, Ctr. Hospitalier Univ. de Rennes (France); P. Jannin, INSERM (France), LTSI, Univ. de Rennes 1 (France); C. Haegelen, INSERM (France), LTSI (France), Ctr. Hospitalier de Rennes (France)

Runner Up: Increasing safety of a robotic system for inner ear surgery using probabilistic error modeling near vital anatomy (9786-51)

Neal P. Dillon, Michael A. Siebold, Jason E. Mitchell, Vanderbilt Univ. (United States), Gregoire S. Blachon, Ramya Balachandran, Vanderbilt Univ. Medical Ctr. (United States), J. Michael Fitzpatrick, Vanderbilt Univ. (United States), Robert J. Webster III, Vanderbilt Univ. Medical Ctr. (United States)

\section{Cum Laude Poster Award}

First Place: Patch-based label fusion for automatic multi-atlas-based prostate segmentation in MR images (9786-72)

Xiaofeng Yang, Ashesh B. Jani, Peter J. Rossi, Hui Mao, Walter J. Curran, Tian Liu, Winship Cancer Institute, Emory Univ. (United States) 